\title{
Numerical position optimization of an over-the-wing mounted engine installation
}

\author{
F. Lange ${ }^{1}$ (D) R. Rudnik ${ }^{1}$
}

Received: 6 January 2020 / Revised: 20 July 2020 / Accepted: 10 November 2020 / Published online: 23 December 2020

(c) The Author(s) 2020

\begin{abstract}
The Collaborative Research Center 880 is investigating different technologies and configurative variants for the purpose of short take-off and landing (STOL) capabilities, ranging from high-lift systems with Coandă flaps to unusual but potentially more efficient engine arrangements. The present study focuses on the reference configuration 3 (REF3). This configuration is characterized by an UHBR over-the-wing nacelle (OWN) located above the wing trailing edge. Starting from the wing/ body configuration the installation effects of the OWN were investigated. A fully automatized surrogate based optimization was used to evaluate the impact of an engine position variation in vertical and horizontal direction to observe fundamental aerodynamic interactions between wing and OWN in cruise flight conditions. Due to the presence of OWN and pylon, a distinct disturbance on the wing upper surface could be observed leading to significant interference effects. Nevertheless, the overall cruise drag of REF3 could be improved by 37 drag counts or nearly $11 \%$ due to the position optimization.
\end{abstract}

Keywords UHBR $\cdot$ CFD $\cdot$ OWN $\cdot$ Optimization $\cdot$ Cruise

\section{List of symbols}

$\begin{array}{ll}\text { AoA } & \text { Angle of attack } \\ c & \text { Mean aerodynamic chord } \\ c_{f} & \text { Friction coefficient } \\ c_{p} & \text { Pressure coefficient } \\ \mathrm{C}_{\mathrm{L}} & \text { Lift coefficient } \\ \mathrm{C}_{\mathrm{D}} & \text { Drag coefficient } \\ \text { dcts. } & \text { Drag counts }(1 \mathrm{dct}=0.0001) \\ \mathrm{h} / \mathrm{c} & \text { Rel. vertical distance } \\ \text { lcts. } & \text { Lift counts }(1 \text { lct. }=0.01) \\ \mathrm{M} & \text { Mach number } \\ \mathrm{OWN} & \text { Over-the-wing nacelle } \\ \mathrm{POT} & \text { Powerful Optimization Tool } \\ \text { Re } & \text { Reynolds number } \\ \mathrm{s} & \text { Wing span } \\ \text { WB } & \text { Wing-body configuration } \\ \text { WBEP } & \text { Wing-body-engine-pylon configuration } \\ \text { x/c } & \text { Rel. horizontal distance }\end{array}$

F. Lange

fabian.lange@dlr.de

1 German Aerospace Center (DLR), Aerodynamics and Flow Technology, Lilienthalplatz 7, 38108 Brunswick, Germany

\section{Introduction}

A number of ambitious goals of the European aeronautical industry and research organizations were defined by the Flightpath 2050 vision [1] in 2011. The stated challenges range from significant fuel burn reductions over further improvements in air safety to strengthening of the European aeronautical industry and research. To address some of the future challenges, a collaboration between the Technical University of Braunschweig, the Leibnitz University of Hannover and the German Aerospace Center (DLR) has been established to form the Collaborative Research Center (CRC) 880. It focuses on multi-disciplinary investigations of innovative technologies by involving different disciplines from aerodynamics over composites to materials including an overall aircraft level assessment.

The investigations focus on aircraft configurations with a capacity of 100 passengers, which feature short take-off and landing (STOL) capabilities including low fuel consumption plus low noise emission. The following study is based on the so-called reference configuration 3 (REF3), illustrated in Fig. 1. This configuration is characterized by a combination of a low wing and an over-the-wing mounted ultra-high bypass ratio (UHBR) engine with a bypass ratio (BPR) of 17. The aircraft should operate at Mach number $M=0.78$ over a mission range of 1100 nautical miles. According to 


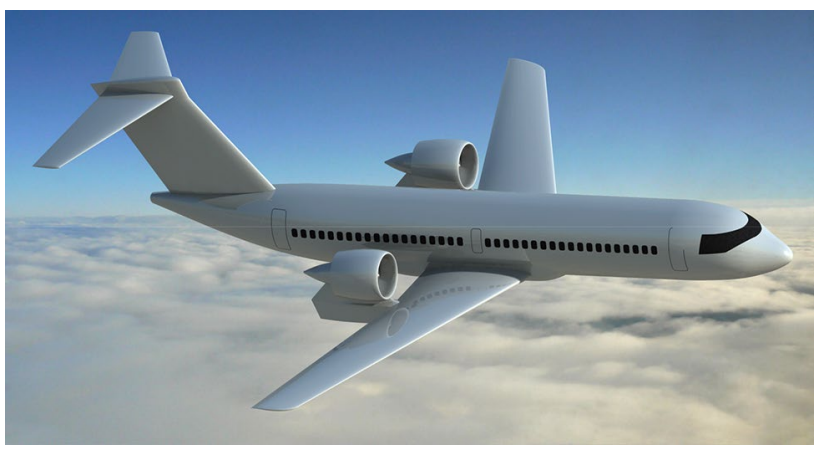

Fig. 1 CRC 880 REF3 configuration [2]

the design cruise condition, the leading edge sweep was set to $26^{\circ}$ to achieve an efficient transonic design. The wing shape is based on the DLR-F15 airfoil [3]. The three-dimensional wing was build based on a preliminary design defined by the PrADO (Preliminary Aircraft Design and Optimization) tool [4]. Furthermore, the STOL performance should comply with an airfield requirement of $900 \mathrm{~m}$. The rationale of this limitation is the connection of small airports located close to residential areas with the existing large airport infrastructure. Therefore, noise emissions need to be reduced significantly. The topics addressed in the CRC 880 range from the investigation of porous materials for noise reduction, for example along the wing's trailing edge [5], to full aircraft design studies considering the potential benefits of an engine installation above the wing. Accordingly, an acoustic shielding of the engines noise emission by the wing can be taken into account [6]. Moreover, a beneficial aerodynamic effect due to the interference between wing suction side and engine inlet can be expected. This was observed by Hooker et al. [7] during an extensive study of different engine positions on commercial aircraft tube and wing configurations.

This article deals with the aerodynamic assessment of the REF3 configuration with the aid of computational fluid dynamics. Therefore, the basic aerodynamic characteristic of the wing-body (WB) configuration will be discussed followed by an evaluation of the interference effects due to nacelle and pylon mounting. Finally, a surrogate-based optimization with the objective of improving the overall drag coefficient by adapting the engine position will be presented.

\section{Wing-body configuration}

As a first step, the WB configuration in cruise flight conditions will be presented to provide information regarding the aircraft's performance and characteristics in the absence of engine components. The grid generation setup will be outlined followed by a grid refinement study. Moreover,
Table 1 Grid characteristics

\begin{tabular}{ll}
\hline Initial layer thickness & $0.0042 \mathrm{~mm}$ \\
& $(0.0001 \%$ \\
& MAC) \\
Stretching ratio (Prisms) & 1.223 \\
Number of prism layers & 44 \\
Number of nodes & $12,694,693$ \\
Number of prisms & $15,588,959$ \\
Number of pyramids & 56,292 \\
Number of tetrahedrons & $24,932,626$ \\
\hline
\end{tabular}

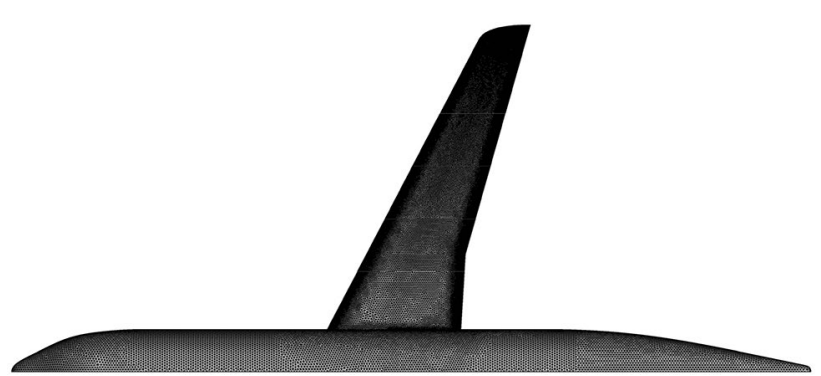

Fig. 2 Surface grid resolution

the CFD parameters will be defined, which were applied throughout the investigation and optimization of REF3.

\subsection{Grid generation}

The grid generation was performed with the aid of the commercial grid generator Centaur by CentaurSoft [8]. This generator enables the creation of hybrid grids, composed of triangles or quadrilaterals on the surface and a near-surface grid based on prisms and hexahedrons. The remaining volume is filled by tetrahedrons. The resolution of the nearsurface grid can be described by first wall spacing, stretching ratio in surface-normal direction and number of layers. Within this study, a local grid refinement was achieved using geometric sources. The grid parameters and properties are listed in Table 1.

An impression of the surface grid and a slice through the volume grid is presented in Figs. 2 and 3.

In addition, a grid convergence study was performed to evaluate the quality of the chosen resolution. The grid resolution study is based on the method proposed by Crippa et al. [9].

Three grid refinement levels were investigated. A coarse and a fine grid have been generated based on the standard medium grid resolution presented in the previous section. A detailed comparison of the grid properties is given in Table 2.

The impact of the grid refinement on the aerodynamic coefficients is outlined in Fig. 4. With respect to the grid 


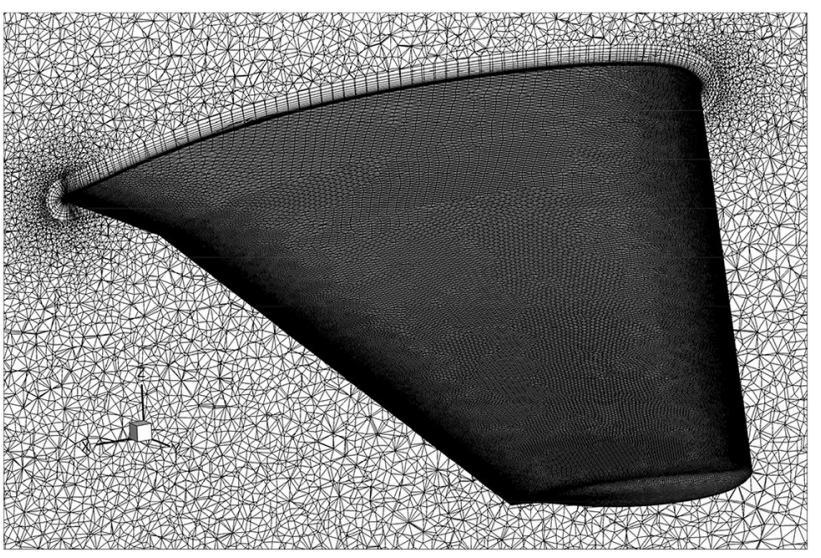

Fig. 3 Detailed view on near-surface grid of the wing

Table 2 Properties of the three grid resolution levels

\begin{tabular}{llll}
\hline & Coarse & Mid & Fine \\
\hline Number of points & $4,072,834$ & $12,694,693$ & $43,356,890$ \\
Initial layer thickness & $0.006 \mathrm{~mm}$ & $0.0042 \mathrm{~mm}$ & $0.0029 \mathrm{~mm}$ \\
Stretching ratio (prisms) & 1.53 & 1.223 & 1.1 \\
Number of prism layer & 22 & 44 & 88 \\
\hline
\end{tabular}

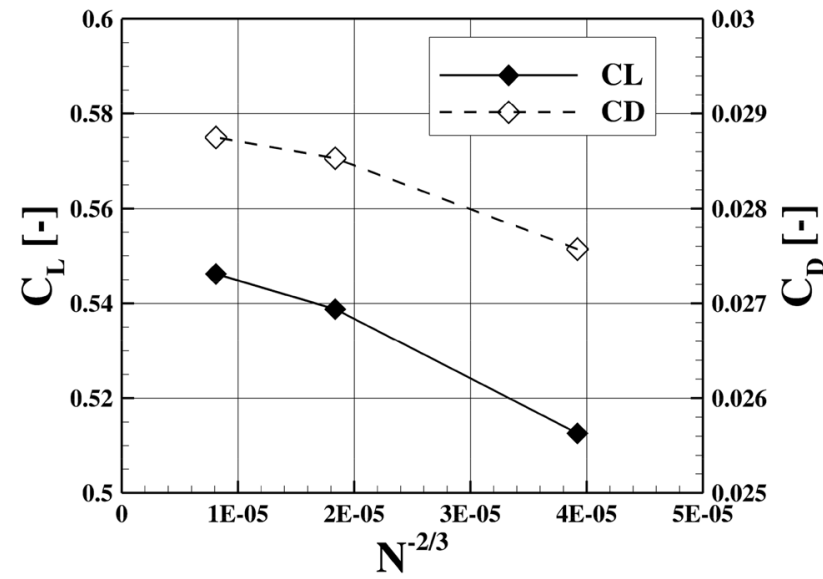

Fig. 4 Lift and drag coefficient of grid resolution study for $\mathrm{AoA}=3^{\circ}$

refinement approach, the lift and drag coefficients are plotted against the grid point number scaled to the power of $(-2 / 3)$. Based on this figure, an assessment of the aerodynamic behavior for the medium grid can be stated. A coarsening of the grid points leads to significantly lower coefficients. However, the refinement of the mid-size grid by a factor larger than 3 with respect to the total point count results in a slight increase of the aerodynamic coefficients $\left(\Delta C_{\mathrm{L}}=0.74\right.$ lcts., $\Delta C_{\mathrm{D}}=2.2$ dcts.). The progression of the curves indicates an asymptotic behavior for an increasing point number $\left(N^{-2 / 3} \rightarrow 0\right)$. With respect to the following optimization, the medium refinement level is considered as sufficient to resolve the aerodynamic properties of this configuration.

\subsection{Numerical setup}

The numerical results were generated with the DLR-TAU Code. This CFD-code is based on an edge-based finite-volume approach solving the compressible three-dimensional Reynolds averaged Navier-Stokes (RANS) equations. The code development started in the mid 1990s within the German CFD project MEGAFLOW [10] and continuously progressed to the present day, mainly driven by the Institute of Aerodynamics and Flow Technology of DLR. The DLR-TAU Code is used in research projects and the European aerospace industry to investigate complex aerodynamic configurations [11]. For the current investigation the Spalart-Allmaras one-equation eddy viscosity model [12] enhanced by the negative formulation (SAN) [13] was applied. Moreover, the spatial discretization was realized by a central scheme in combination with the implicit LUSGSscheme for time stepping. The artificial dissipation is composed of a combination of matrix $(60 \%)$ and scalar $(40 \%)$ dissipation. Finally, a $2 \mathrm{~W}$-multigrid cycle was selected to achieve convergence acceleration. In the following, all computations were performed at cruise conditions at $M=0.78$ and $\operatorname{Re}=21.5 \times 10^{6}$ based on a mean aerodynamic chord $c=3.805 \mathrm{~m}$.

\subsection{WB evaluation}

Based on the medium grid level, the aerodynamic properties of the WB configuration will be discussed. The evaluation will be carried out at $C_{\mathrm{L}}=0.469$, which corresponds to the maximum landing weight of about $43.9 \mathrm{t}$. operating at $M=0.78$ in an altitude of $37000 \mathrm{ft}$. The required lift results in an $\mathrm{AoA}=2.43^{\circ}$ and $C_{\mathrm{D}}=0.02512$. The wing is characterized by a reference area of $99 \mathrm{~m}^{2}$ and a wing span of $28.745 \mathrm{~m}$ plus a leading edge sweep of $26^{\circ}$. The distribution of the pressure coefficient $c_{\mathrm{p}}$ on the upper and lower surface is plotted in Fig. 5.

Based on this visualization, the shock position (white) on the rear upper wing can be detected. Moreover, a preferred parallel alignment of the iso- $c_{\mathrm{p}}$ values in between wing leading and trailing edge was achieved. At the leading edge at $y / s=50 \%$, locally higher pressure coefficients can be observed, which leads to a bump in the chordwise $c_{\mathrm{p}}$ distribution, also shown in Fig. 6. Nevertheless, the shock position is aligned parallel to the trailing edge along the wing span. On the lower wing surface, the rear loading close to the trailing edge can be identified (green). Besides the areal impression of the wing's pressure distribution, more details can be gathered from four slices extracted at $20 \%$, 40\%, 60\% and 80\% wingspan, shown in Fig. 6. 


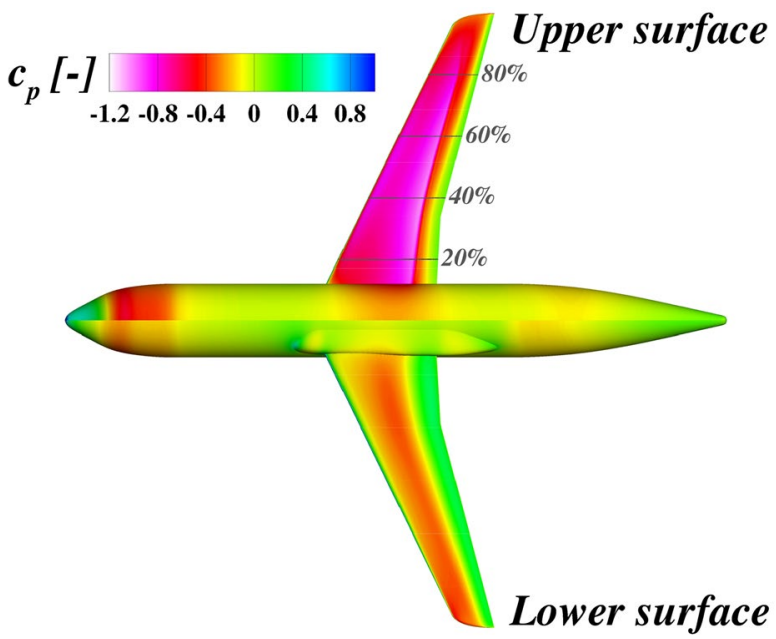

Fig. $5 c_{\mathrm{p}}$ distribution on WB from top and bottom view

Based on these four slices, a typical transonic pressure distribution can be detected. The wing shape is based on the DLR F-15 airfoil, which was extracted from the FNG wing [3]. First, the pressure distribution at $y / s=60 \%$ will be discussed. Along the suction side, a strong decrease of $c_{\mathrm{p}}$ can be detected at the leading edge up to $c_{\mathrm{p}}=-0.8$ resulting in local supersonic flow. The critical pressure coefficient $c_{\mathrm{p} \text {,crit }}$ defining the transition from subsonic to supersonic flow is defined by $c_{\mathrm{p}, \text { crit }}=0.494$ for $M=0.78$. In the region $0.1<x / c<0.6$ the gradients of $c_{\mathrm{p}}$ are small and the pressure level changes not more than \pm 0.15 . This segment of low pressure ends with a strong gradient, indicating the shock position. The appearance of the pressure distribution is comparable with a rooftop pressure distribution. The pressure distribution on the lower wing is dominated by a rear loading in the aft wing section.

At $y / s=40 \%$, the pressure distribution is comparable with the previous observations. Solely a slight downstream shift of the shock position can be detected for the section located further inboard.

The most inboard pressure distribution at $y / s=20 \%$ is following this trend. The shock is located at $x / c=0.8$. Moreover, a higher pressure value at the leading edge can be detected, which decreased along the chord till the shock occurs. This pressure increment along the leading edge between the pressure distributions at $y / s=20$ and $40 \%$ can be ascribed to the three-dimensional effects originating from the wing/fuselage junction.

Another three-dimensional effect can be observed close to the wing tip at $y / s=80 \%$. The shock position is shifted significantly further upstream due to end effects of the wing. In addition, the higher $c_{\mathrm{p}}$-values along the suction side are indicating a reduced aerodynamic loading of the
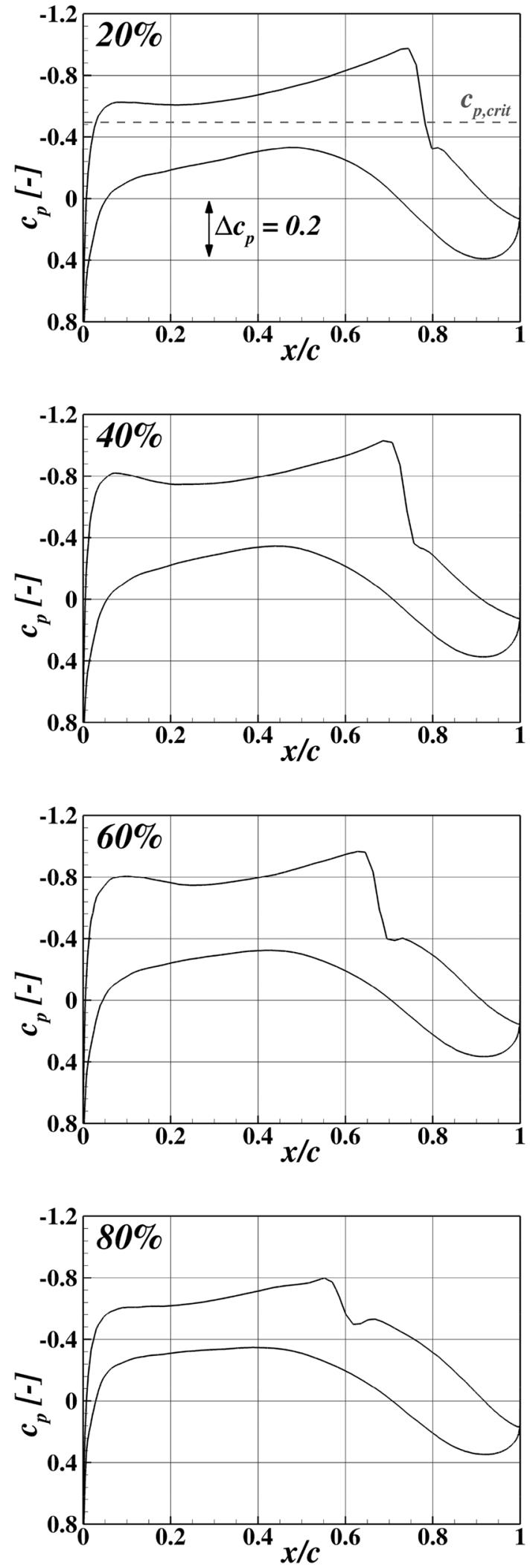

Fig. 6 Pressure distribution at four spanwise positions 
Table 3 Engine setting

\begin{tabular}{lll}
\hline & Bypass & Core \\
\hline$T_{\mathrm{t}} / T_{\mathrm{t} 0}$ & 1.102 & 2.552 \\
$p_{\mathrm{T}} / p_{0}$ & 2.021 & 1.470 \\
\hline
\end{tabular}

outboard wing compared with the inboard section, due to the predefined twist distribution.

In general, the transition from supersonic to subsonic speed, especially for the inboard wing, is associated with a shock with a $\Delta c_{\mathrm{p}}$ up to 0.6 . This indicates a strong shock resulting in high drag values.

An analysis of the far-field drag with the aid of the ONERA tool ffd00 [14] provides further information regarding the drag components. Nearly $40 \%(\sim 100$ dcts.) of the overall drag is generated by friction. About $10 \mathrm{dcts}$. are assigned to wave drag, which supports the earlier statement with respect to the strong shock. The remainder is dominated by viscous pressure ( $\sim 43 \mathrm{dcts})$ and induced drag ( $97 \mathrm{dcts})$.

\section{Impact of engine installation}

In the following section, the impact of the installation of engine and pylon will be analyzed and compared to the aerodynamic performance of the WB configuration. As mentioned before, the engine will be located above the wing close to the wing trailing edge as defined in the REF3 Data Sheet [15] at a spanwise position of $y / s=0.314$. The grid generation was performed based on the setup defined in Sect. 2.1. Moreover, the comparison between WB and Wing/Body/Engine/Pylon (WBEP) configuration will be performed at identical lift coefficients $C_{\mathrm{L}}$. The engine conditions were simulated based on a previous design study with GasTurb [16]. The pressure and temperature ratios for the outlet conditions for the UHBR engine jets are listed in Table 3. The inlet mass-flow is coupled with the exhaust mass-flow, which is derived from the core and fan jet flow according to the defined engine settings.

The resulting thrust nearly equals the overall drag of the WBEP configuration, which results in steady flight conditions. An investigation by Ahuja et al. [17] has shown that the interaction for an OWN configuration is dominated by the influence of the wing on the engine, but not vice versa. Thus, the difference between thrust and drag of about $1 \%$ justifies a constant thrust setting for an aerodynamic evaluation.

The overall drag at target lift $C_{\mathrm{L}}=0.465$ totals $351 \mathrm{drag}$ counts (dcts.) at $\mathrm{AoA}=3.924^{\circ}$. Compared to the WB configuration, an increase of 100 drag counts can be ascertained due to the engine installation. To identify the source of this significant drag rise, a detailed analysis is carried out to reveal, whether the engine, engine position or the

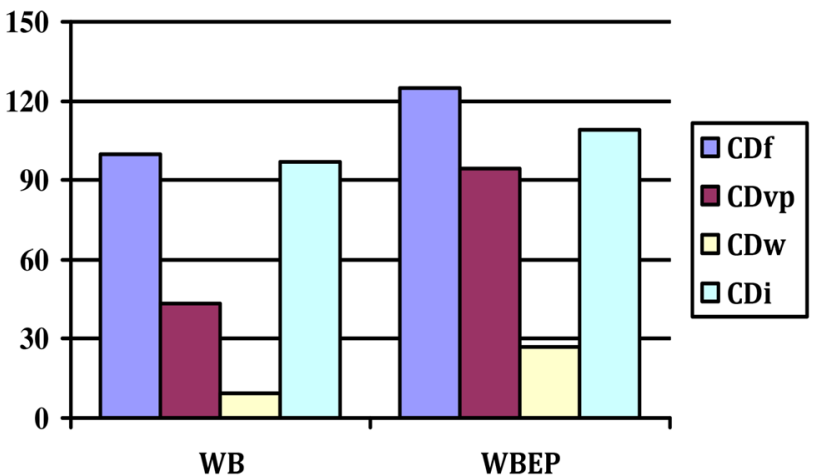

Fig. 7 Comparison of drag components between WB and WBEP

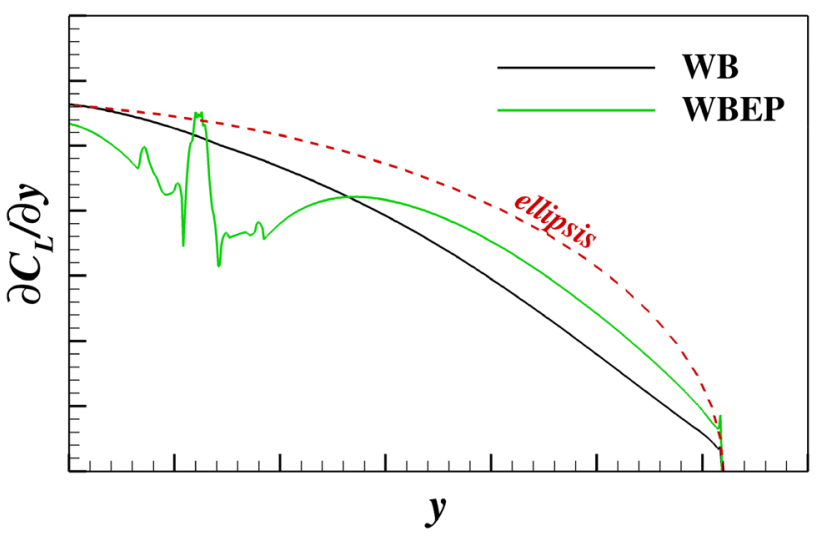

Fig. 8 Spanwise lift distribution for WB and WBEP

large pylon is responsible for this significant degradation of the aircraft's performance. First, an evaluation of the drag components with ffd00 provides some indication. The diagram in Fig. 7 compares the drag components of the WB and WBEP configuration. Based on this figure, an increase in each individual drag component due to the engine integration can be observed.

However, the largest relative increment can be assigned to the viscous pressure (CDvp) ${ }^{1}$ and wave drag (CDw) component. The friction drag (CDf) increment can be explained by the increase of the wetted area by about $27 \%$ due to the installation of pylon and nacelle. The increase of induced drag (CDi) is based on the disturbance of the spanwise lift distribution due to the engine installation and the associated higher AoA to reach the target lift coefficient. The impact of the engine installation on the spanwise lift distribution is presented in Fig. 8. Thus, the origin of an increased viscous pressure and wave drag needs to be identified. Therefore, a

\footnotetext{
${ }^{1}$ Drag component originating from a shape modification due to an emerging boundary layer resulting in additional pressure drag.
} 


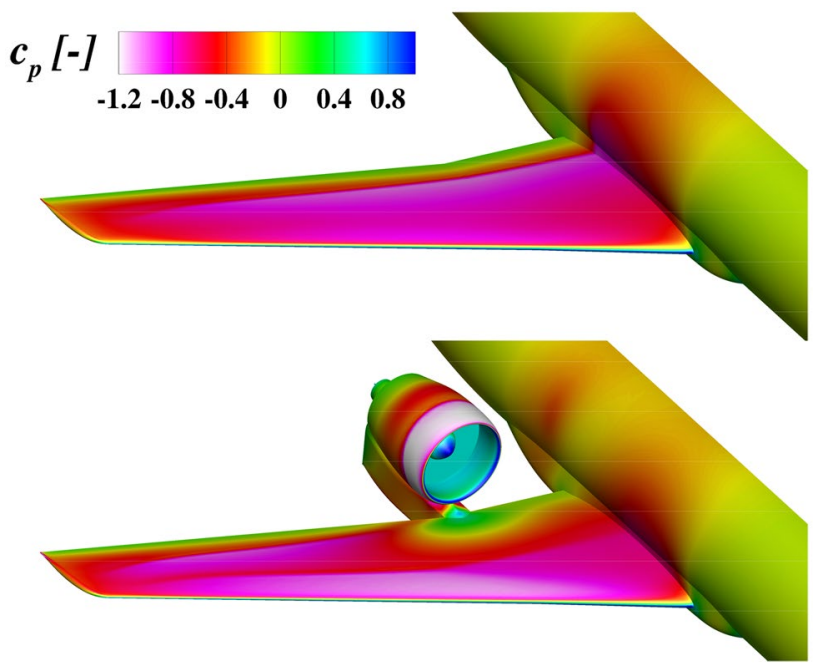

Fig. 9 Comparison of $c_{\mathrm{p}}$-distribution for WB and WBEP

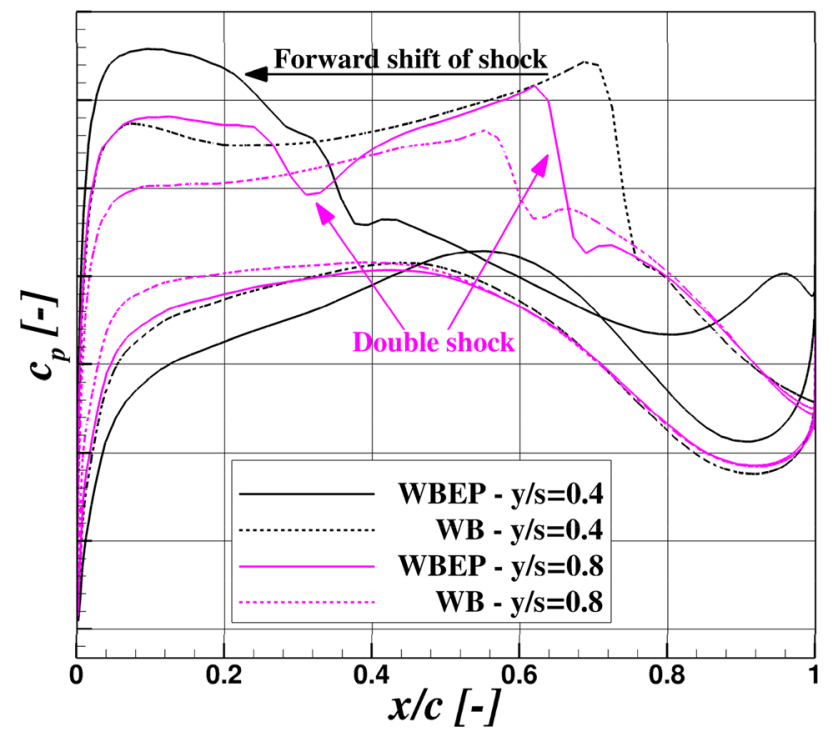

Fig. $10 c_{\mathrm{p}}$-distribution at $y / s=0.4$ and $y / s=0.8$ comparing $\mathrm{WB}$ and WBEP

comparison of WB and WBEP configuration at identical $C_{\mathrm{L}}$ is presented in Figs. 9 and 10.

The impact of the engine installation at the wing trailing edge on the wing aerodynamic is substantial. Based on the surface $c_{\mathrm{p}}$-distribution in Fig. 9 and the chordwise pressure distributions at $y / s=40 \%$ and $80 \%$ in Fig. 10, an alteration of the shock position and propagation can be observed.

For the WB configuration, the shock is positioned within the last quarter of the chord length. In contrast, due to the engine installation, the shock along the inboard wing section is shifted significantly upstream for identical $C_{\mathrm{L}}$ and a double shock structure on the outboard wing can be observed.

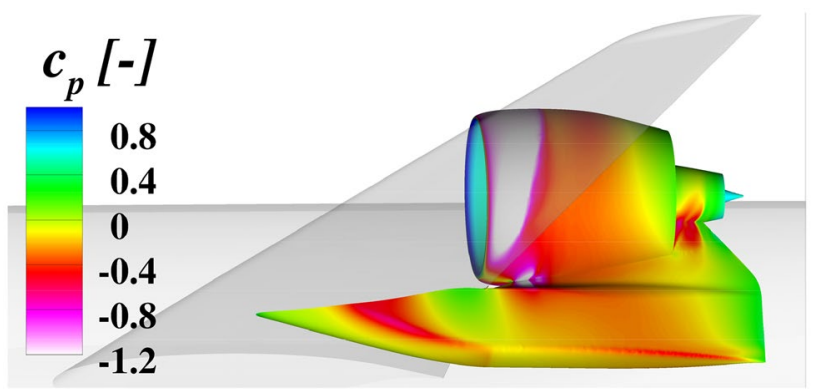

Fig. 11 Bottom view on nacelle/pylon/wing junction (airframe is transparent)

Moreover, a large supersonic area on the fore-body of the nacelle can be detected. Taking these effects into account, the increase of wave drag due to the engine installation can be explained. Furthermore, the reduction of the low pressure area on the inboard wing results in higher AoA $=3.92^{\circ}$ for the target $C_{\mathrm{L}}$. This region of higher $c_{\mathrm{p}}$ in front of the engine originates from the engine inlet, which reduces the flow velocities in the inlet streamtube before entering the engine. Furthermore, the view from the bottom on the junction between pylon and nacelle in Fig. 11 reveals a disturbance of the low pressure region at the nacelle leading edge. This low pressure region on the forward facing surface is beneficial with respect to drag, because it results in a thrust component. However, due to the intersection of pylon and nacelle, this effect is reduced. Furthermore, high pressure coefficients are visible on the pylon, close to the wing trailing edge in Fig. 9. At this junction, an additional pressure drag component is generated due to the upstream facing pylon surface.

In summary, the OWN installation results in a significant drag rise of about 100 drag counts. Especially, wave and viscous pressure drag are increased disproportional. The related drag sources were identified. Next, the overall aircraft performance will be improved by optimizing the engine position to reduce these interference effects.

\section{Engine position optimization}

The following section deals with the optimization of the OWN position. In Sect. 3, the impact of the engine installation has been discussed and potential drag sources have been identified. However, the significant drag increase of about 100 drag counts between the WB and WBEP configuration indicates a substantial margin for improvement. Therefore, an optimization has been set up to reduce the overall drag coefficient of the WBEP configuration by optimizing the engine position. However, the position variation is limited by two constraints. On the one hand, the overlap between 
Fig. 12 Parameterized geometry for engine position variation

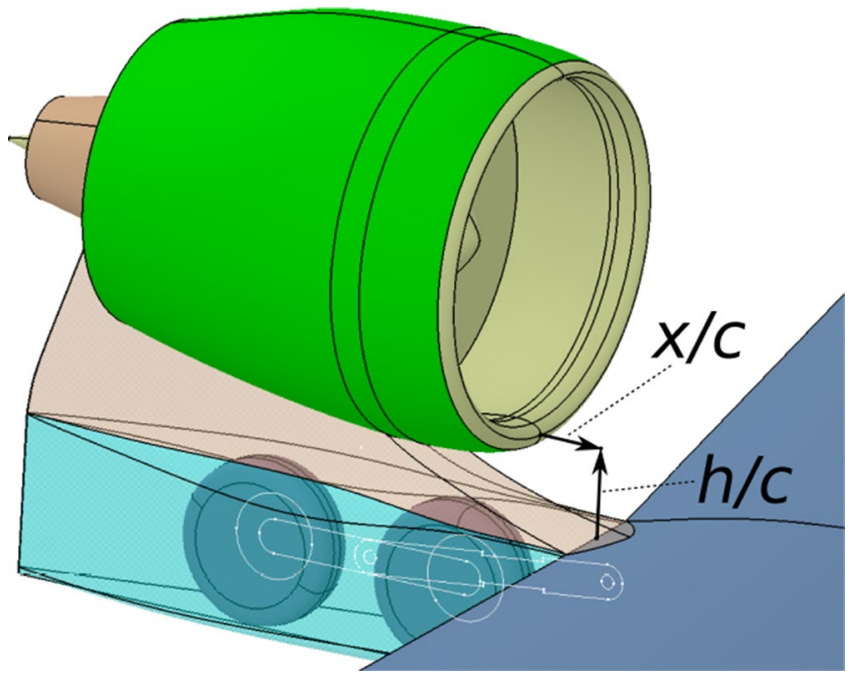

Cauchy convergence control criterion was applied during the optimization. A maximum deviation of 0.1 drag counts in 750 iterations leads to a termination of the calculations. This criterion ensures a high quality as well as comparability of each design iteration. The identification of the exact aerodynamic coefficients is obtained by the drag prediction tool AeroForce [18]. It is based on the evaluation of surface data. Additionally, corrections of the engine's pre-entry and post-exit drag are taken into account. This is achieved by a separation of airframe drag and thrust components due to the localization of the stagnation line along the nacelle inlet.

Ensuing, the aerodynamic coefficients are processed by the in-house optimization tool "Powerful Optimization Tool with Surrogate Modelling" (POT). A detailed description of POT is given in Sect. 4.2. Finally, the next set of parameters for the subsequent iteration is provided by the optimization tool.

\subsection{Optimization tool POT}

The optimization was driven by two objectives. On the one hand, an optimized engine position should be identified, which is characterized by minimum drag. On the other hand the impact of different engine positions for an OWN configuration should be explored. Therefore, the optimization framework POT was chosen, which focuses on surrogate based optimizations. It was compiled by Wilke [19] to obtain a versatile environment to solve various problems without any bond to a certain CFD solver or grid generator. Moreover, a wide range of different algorithms and techniques for direct, single or variable-fidelity surrogate based optimization is implemented. This investigation utilizes a single fidelity surrogate based optimization. The process can be subdivided into three components: 


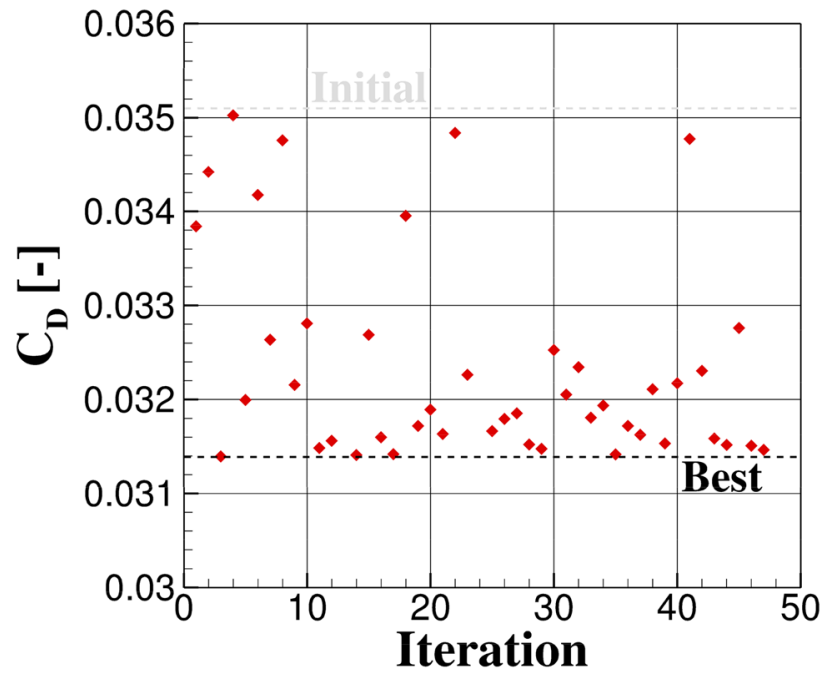

Fig. 13 Optimization history

1. Design of experiments (DoE).

2. Surrogate models.

3. Optimization strategy.

Initially, a design of experiments needs to be performed to explore the parameter space by a defined number of samples. POT offers several algorithms for a uniform sample distribution, which is important for the approximation based on the first data base, resulting in the initial surrogate model. For this case, an advanced Latin Hypercube, the Central Voronoi Tessellation (CVT) was selected.

The surrogate model is generated with the aid of a Kriging model [20]. Kriging is a combination of a trend function and radial basis function, which enables a sensible approximation of the goal function dependent on the sampling. To achieve a robust, efficient and accurate utilization of the approximation model, several settings, like regression model or treatment of numerical noise, can be adapted to align the surrogate model with the actual physical behavior. Then, the optimization is started from the initial surrogate model. However, in this case, the optimizer is not only targeting an optimum, but also improving the surrogate model by the application of an adaptive sampling strategy. Therefore, a hybrid optimization approach was used separated into a differential evolution (DE) algorithm and a gradient-free Simplex pattern search method. Due to this procedure, global regions of interest will be identified by the $\mathrm{DE}$, whereas the pattern search determines the local optimum in these regions more accurate.

\subsection{Evaluation}

The optimization was initialized with ten samples for the DoE, to conform a minimum guide number of five samples

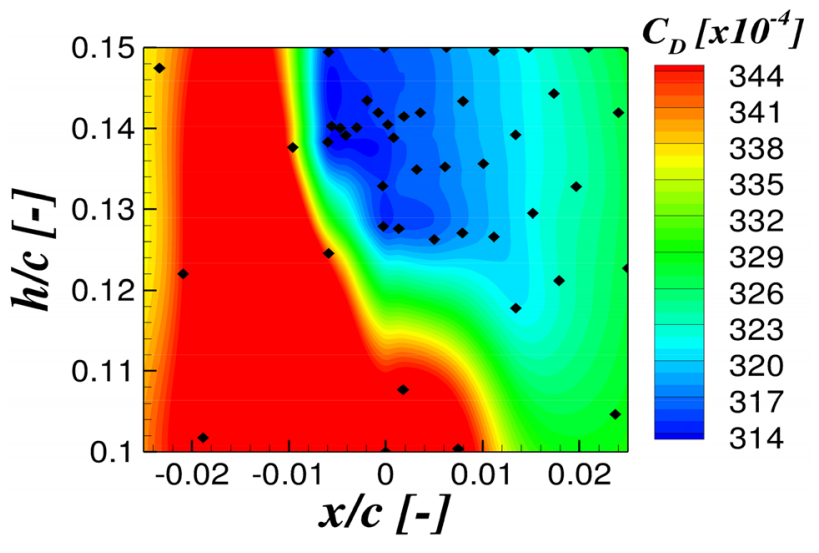

Fig. 14 Final surrogate model

per parameter. Based on this DoE data points, an initial surrogate model was created. The subsequent optimization required 37 additional iterations to achieve a sufficient design confidence for the surrogate model. The history of the optimization process is plotted in Fig. 13.

The graph indicates that within the DoE, one parameter combination was already close to the optimum. Nevertheless, a large number of iterations were necessary to resolve the behavior of the objective function within the parameter space. The reason behind this costly sampling becomes clear when the final surrogate model is examined.

The surrogate model is presented in Fig. 14 The vertical distance $\mathrm{h} / \mathrm{c}$ between nacelle leading edge and wing trailing edge is plotted over the horizontal distance $x / c$. Positive values for $x / c$ imply an overlap of nacelle and wing. The contour plot represents the objective function $C_{\mathrm{D}}$ dependent on the position parameters. Red areas are detrimental and imply high drag values, blue symbolizes beneficial areas of low drag. Based on this contour plot, a steep transition between the beneficial and detrimental area is obtained for $-0.01<x / c<0$ for medium to high $h / c$ values. Because of these high gradients, the optimizer added several additional points, recognizable by the black dots, to improve the approximation. The sampling of the beneficial area displays a uniform distribution. The area of high drag values is not investigated in more detail by POT. Generally, an engine position for $h / c>0.125$ and an overlap of $0<x / c<0.015$ can be considered as favorable with respect to the overall drag coefficient for REF3.

Next, the reason for the behavior of the objective function will be investigated in more detail. Therefore, the drag contribution of each aircraft component was evaluated with the aid of the surface-based drag analysis tool AeroForce. Due to this evaluation, distinct variations of the drag coefficient were observed for pylon, wing, and nacelle. The influence of vertical and horizontal distance 


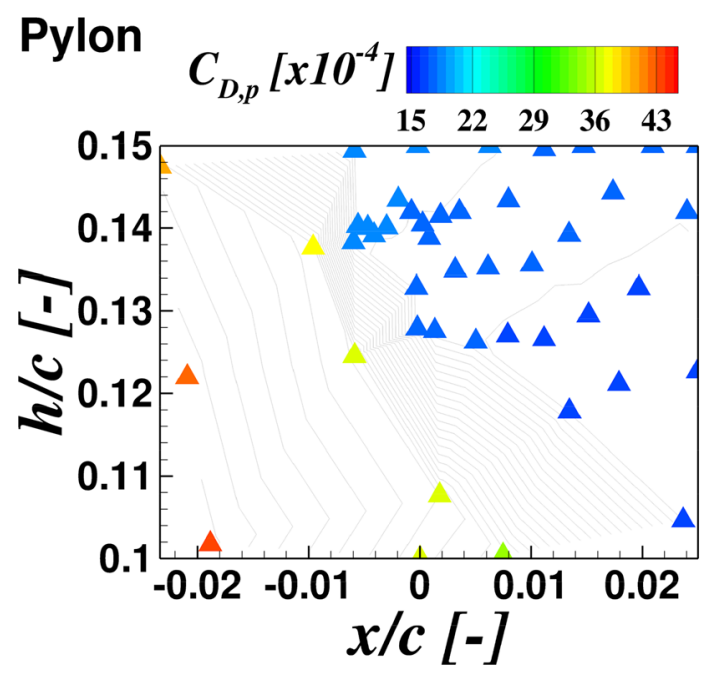

Nacelle

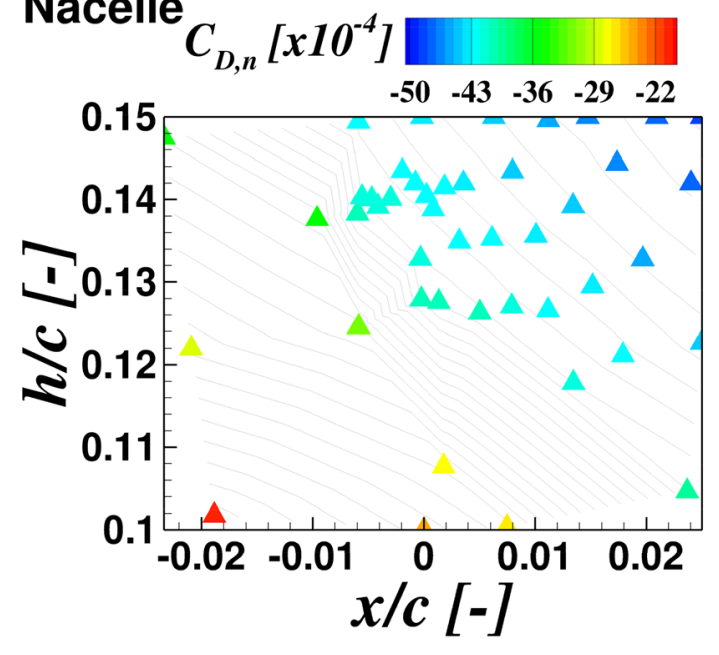

Wing

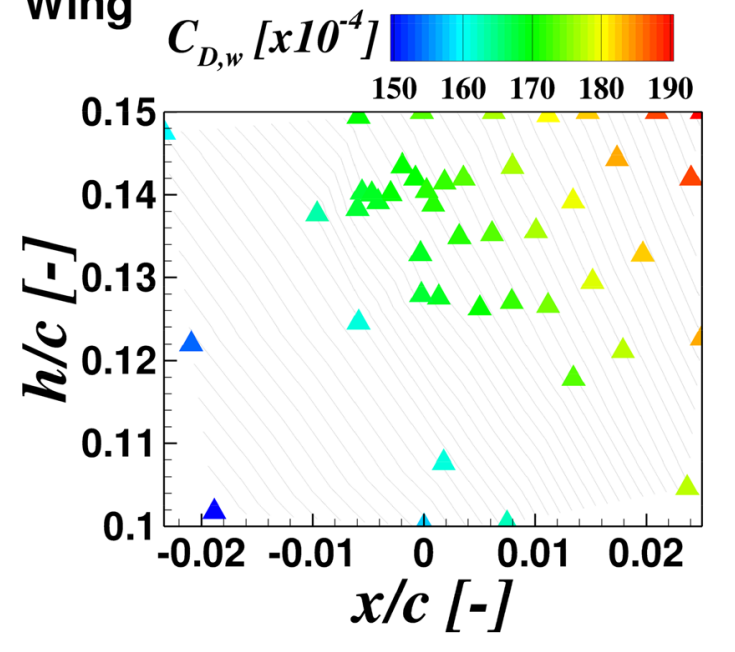

Fig. 15 Influence of engine position on component-wise drag coefficient for pylon $C_{\mathrm{D}, \mathrm{p}}$, nacelle $C_{\mathrm{D}, \mathrm{n}}$ and wing $C_{\mathrm{D}, \mathrm{w}}$

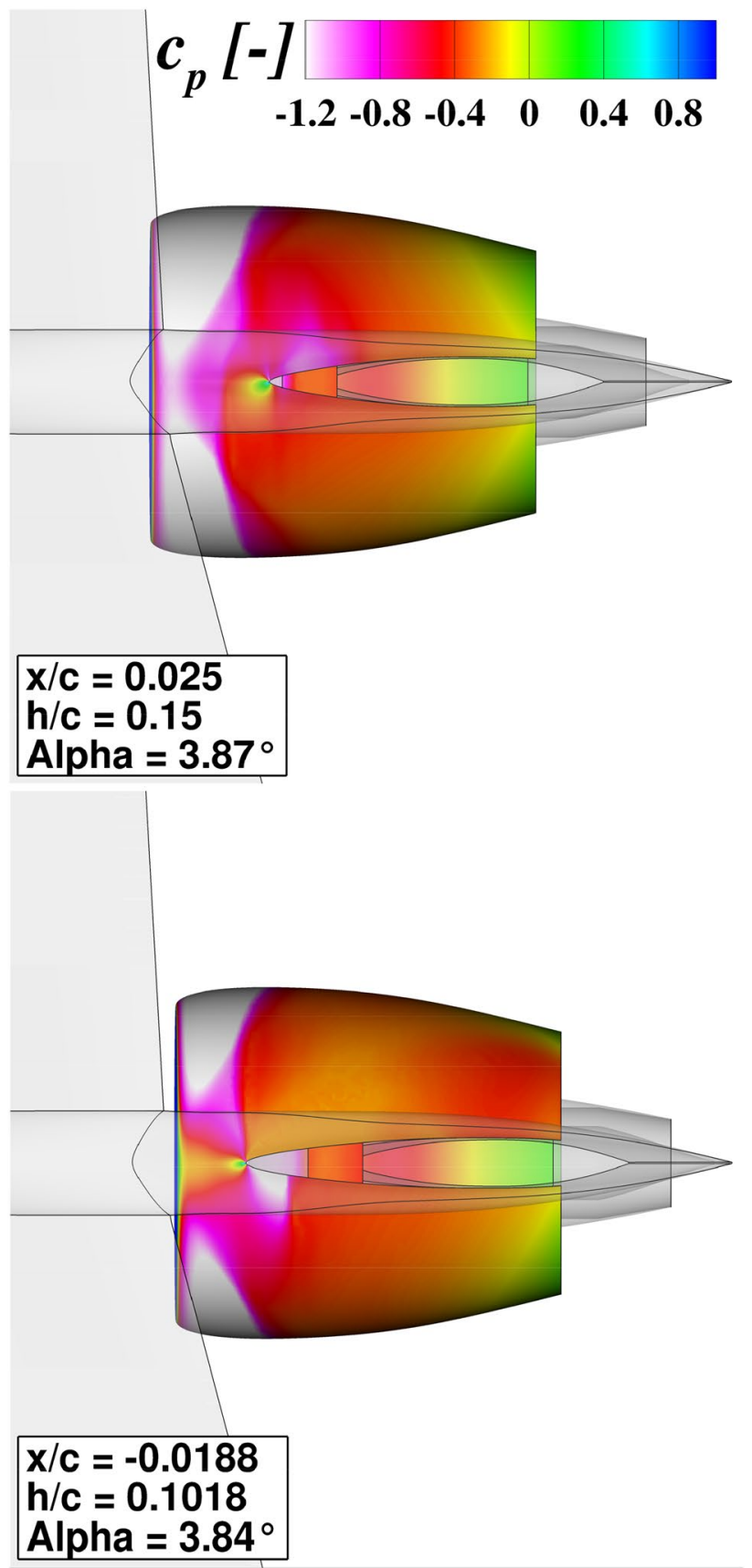

Fig. 16 Influence of horizontal and vertical distance on nacelle leading edge

on the component-wise drag coefficient is plotted in Fig. 15.

These plots are revealing the origin of the non-linear behavior of the overall drag coefficient. For both, pylon and nacelle, a steep drag increase can be observed for $x / c \sim 0$. The wing behavior is constantly rising, however the difference between the lower left and upper right parameter space is about 40 drag counts. Consequently, $C_{\mathrm{D}, \mathrm{w}}$ is rising with overlap and increasing vertical distance between wing 


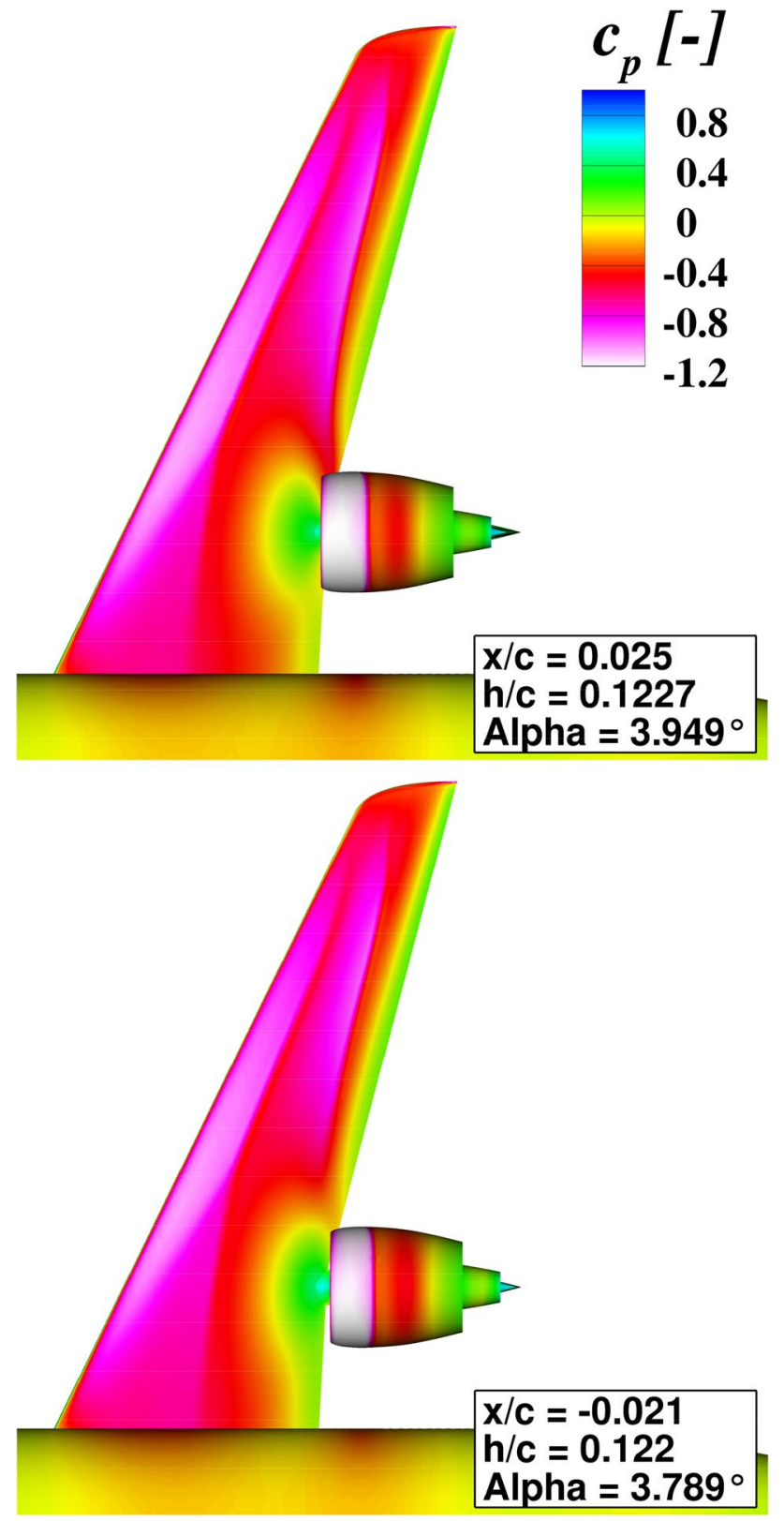

Fig. 17 Influence of horizontal distance $x / c$ on wing upper surface

and nacelle. However, the linear wing behavior is superimposed by a strong effect occurring on pylon and nacelle. For these components, a shift of the nacelle leading edge behind the wing trailing edge results in a sudden and dominant drag increase. As a consequence, the optimum engine position can be found at $x / c=-0.005$ and $h / c=0.138$ with $C_{\mathrm{D}}=0.0314$. Nevertheless, the aerodynamic interference effects, which are leading to this optimum, should be further studied. Therefore, the numerical solution of several iterations, extracted from the optimization process, will be investigated and compared in more detail.
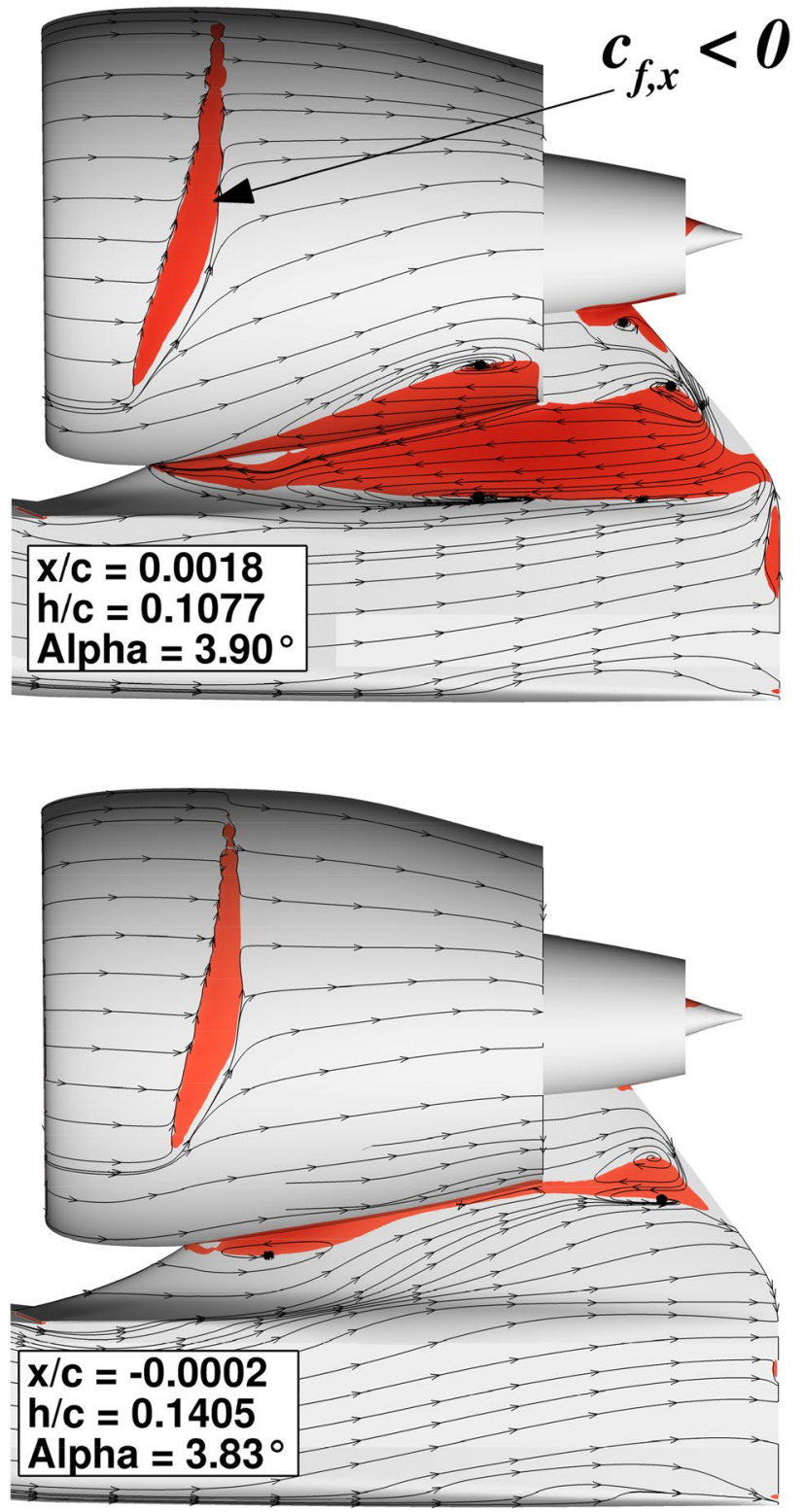

Fig. 18 Influence of the vertical distance $h / c$ on nacelle and pylon

The influence of the junction between nacelle and pylon is presented in Fig. 16. Based on the pressure coefficient $c_{\mathrm{p}}$, the flow distortion due to the pylon can be noticed. On the lower figure, an engine position further downstream and with reduced vertical distance leads to an increasing $c_{\mathrm{p}}$ value along the nacelle leading edge. Thus, the beneficial interference effect of the over-the-wing mounted nacelle in the region of high velocities above the wing is reduced. In contrast, the upper figure reveals an undisturbed low pressure region at the nacelle leading edge for an engine position with maximum overlap and maximum vertical distance. Consequently, an overlap of nacelle leading edge and wing trailing 
edge is beneficial. Larger vertical distances are preferable to improve the junction between nacelle and pylon. Positions further downstream result in a decrease of the interference effects around the pylon intersection. This finding corresponds with Fig. 15.

However, the overlap between nacelle and wing also affects the complete wing aerodynamics. This is shown in Fig. 17. Based on the $c_{\mathrm{p}}$-distribution, the double shock characteristic due to the OWN installation can be identified for both configurations. Nevertheless, a larger extent of the second shock on the outer wing can be noticed because of an increased overlap for a comparable vertical distance in the upper plot. As a consequence, higher wave drag values can be obtained and the supersonic region above the outer wing interacts with the nacelle shock followed by a large shock induced separation. Consequently, an engine position further downstream is beneficial with respect to the wing aerodynamics. This finding aligns as well with the evaluation in Fig. 15.

Finally, the influence of the vertical distance on nacelle and pylon is presented in Fig. 18. The plot visualizes the wall shear stress coefficient $c_{\mathrm{f}}$ evaluated by surface streamlines and in addition areas with $c_{\mathrm{f}, \mathrm{x}}<0$ as a contour plot on the inboard surfaces of nacelle and pylon. On the lower part of Fig. 18, the engine is positioned close to the maximum $h / c$-position. For this iteration, especially a shock induced flow separation on the nacelle can be noticed by the red colored region. On the upper figure, the nacelle is located at a similar $x / c$-position but nearly at minimal vertical distance. Due to this close coupling, besides a small flow separation on the nacelle, a large flow separation occurs at the lower nacelle and pylon, noticeable by the red color. This can be explained by the reduced channel between wing upper side and engine streamtube, which detains high energy flow from filling up the junction between nacelle and pylon. Thus, the weak flow is not able to follow the contour and separates locally from the surface, which leads to a drag increase. Based on this observation, an engine position at larger vertical distances should be preferred. Otherwise, a drag increase is to be expected because of large flow separations on pylon and nacelle.

\section{Conclusion}

Concluding, the installation of an OWN engine results in complex aerodynamic interferences. The fixed pylon shape adds in this particular case a strong design limitation. The interaction between OWN and wing upper shape results in a formation of a double shock on the outer wing due to a significantly increased angle of attack and a distinct impact on the inboard wing loading. A beneficial interaction between the high velocity flow above the wing and nacelle leading edge could be observed. The variation of vertical and horizontal distance revealed a complex interference between different effects. A separated evaluation of the aircraft components within the investigated parameter space enabled a detailed identification of the beneficial and detrimental interference effects. In general, an engine position with a slight overlap of nacelle highlight and wing trailing edge is preferable. Due to this positioning, the beneficial interaction between nacelle leading edge and wing is not disturbed by the pylon junction and the impact on the outer wing flow is tolerable. Moreover, the vertical distance between nacelle and wing should be increased to prevent flow separations on pylon and lower nacelle but simultaneously allow an optimal interference to achieve the low pressure area on the nacelle leading edge. Finally, the engine position optimization resulted in an overall improvement of $37 \mathrm{dcts}$. compared with the reference configuration. However, the adaptation of the engine position was the first step to exploit the potential of an OWN configuration. Subsequently, the wing shape should be adapted to suppress for instance the high wave drag component induced by the double shock on the outer wing.

Acknowledgements Financial support has been provided by the German Research Foundation (Deutsche Foschungsgemeinschaft-DFG) in the framework of the Sonderforschungsbereich 880 . In the design of the geometry of the reference configuration and in the solution of many technical issues, Arno Ronzheimer from the Transport Aircrafts department of DLR Braunschweig gave a fundamental and really valuable support. The authors want to acknowledge Gunther Wilke for the support given in the optimization procedure and ONERA for providing the ffd00 software for far-field drag decomposition utilized for this research.

Funding Open Access funding enabled and organized by Projekt DEAL.

Open Access This article is licensed under a Creative Commons Attribution 4.0 International License, which permits use, sharing, adaptation, distribution and reproduction in any medium or format, as long as you give appropriate credit to the original author(s) and the source, provide a link to the Creative Commons licence, and indicate if changes were made. The images or other third party material in this article are included in the article's Creative Commons licence, unless indicated otherwise in a credit line to the material. If material is not included in the article's Creative Commons licence and your intended use is not permitted by statutory regulation or exceeds the permitted use, you will need to obtain permission directly from the copyright holder. To view a copy of this licence, visit http://creativecommons.org/licenses/by/4.0/.

\section{References}

1. Flightpath 2050: Europe's vision for aviation. Publication Office of the European Union, Luxembourg (2011)

2. Savoni, L., Rudnik, R.: Aerodynamic Assessment of PylonMounted Over-the-Wing Nacelle Installations on a STOL 
Commercial Aircraft Concept. New Results on Numerical and Experimental Fluid Mechanics. Springer, Berlin (2016)

3. Dargel, G., Hansen, H., Wild, J., Streit, T., Rosemann, H., Richter, K.: Aerodynamische Fluegelauslegung mit Multifunktionalen Steuerflächen. DGLR-2012-096 (2002)

4. Heinze, W.: Ein Beitrag zur quantitativen Analyse der technischen und wirtschaftlichen Auslegungsgrenzen verschiedener Flugzeugkonzepte für den Transport grosser Nutzlasten. ZLR-Forschungsbericht 94-01. ISBN 3-928628-14-3 (1994)

5. Ananthan, V.B., Akkermans, R.A.D., Bernicke, P., Ewert, R., Dierke, J., Rossian, L.: Investigation of effect of porous material on trailing edge noise by overset-LES. In: ETMM12, Montpellier, France (2018)

6. Blinstrub J., Bertsch L., Heinze W.: Assessment of the noise immission along approach and departure flightpaths for different SFB 880 vehicle concepts. In: 2018 AIAA/CEAS Aeroacoustics Conference, AIAA paper 2018-2818. https://doi. org/10.2514/6.2018-2818 (2018)

7. Hooker, J., Wick, A., Zeune, C., Agelastos, A.: Over-the-wing nacelles installations for improved energy efficiency. AIAA paper 2013-2920 (2013)

8. CentaurSoft: Centaur Hybrid Grid Generation System. (2018). http://www.centaursoft.com. Accessed 10 Sept 2018.

9. Crippa, S., Melber-Wilkending, S., Rudnik, R.: DLR Contribution to the first high lift prediction workshop. AIAA paper 2011-938 (2011)

10. Gerhold, T.: Overview of the Hybrid RANS Code TAU. In: Kroll, N., Fassbender, J. (eds.) MEGAFLOW-Numerical Flow Simulation for Aircraft Design. Notes on Numerical Fluid Mechanics and Multidisciplinary Design, NNFM, vol. 89, pp. 81-92. Springer, Heidelberg (2005)

11. Rudnik, R., Melber-Wilkending, S., Risley-Settle, P.: TAUSOLAR Contribution to the 3rd high lift prediction workshop. AIAA paper 2018-1035 (2018)
12. Spalart, P.R., Allmaras, S.R.: A one-equation turbulence model for aerodynamic flows. AIAA paper 92-0439 (1992)

13. Allmaras, S.R., Johnson, F.T., Spalart, P.R.: Modification and clarification for the implementation of the Spalart-Allmaras turbulence model. In: 7th International Conference on Computational Fluid Dynamics, ICCFD7-1902 (2012)

14. Destarac, D.: Far-field/near-field drag balance and applications of drag extraction in CFD. CFD-based aircraft drag prediction and reduction lecture series, pp. 1-65. Von Karman Inst. for Fluid Dynamics, Hampton (2003)

15. Heinze, W., Weiss, T.: Main data sheet. A/C Type: SFB 880 Reference Aircraft REF3-2015 (2015)

16. GasTurb: GasTurb GmbH. (2018). http://www.gasturb.de/. Accessed 10 Sept 2018

17. Ahuja, J., Renganathan, A., Berguin, S., Mavris, D. N.: Multidisciplinary analysis of aerodynamics-propulsion coupling for the OWN concept. In: 2018 Multidisciplinary Analysis and Optimization Conference, AIAA paper 2018-2927. https://doi. org/10.2514/6.2018-2927 (2018)

18. Wild, J.: AeroForce - thrust/drag bookkeeping and aerodynamic force breakdown over components update v 0.6.8. DLR internal report, DLR-IB-AS-BS-2018-51 (2018)

19. Wilke, G.A.: Aerodynamic optimization of helicopter rotor blades using variable fidelity methods. Ph.D. Thesis, DLR (German Aerospace Center) (2017)

20. Krige, D.: A statistical approach to some mine valuation and allied problems on the Witwatersrand. Ph.D. Thesis, University of the Witwatersrand (1951)

Publisher's Note Springer Nature remains neutral with regard to jurisdictional claims in published maps and institutional affiliations. 